\title{
Effects of Resistance Exercise and Fermented Soybean Consumption on Glucose Tolerance and Expressions of Immune Senescence-Related Myokines in Middle-Aged Obese Rats
}

\author{
Kijin Kim ${ }^{1, *}$, Nayoung Ahn'1, Suryun Jung' ${ }^{1}$ Youngsik Ju', Gyuho Lee', Miyeon Kim², Yongjin Jeong ${ }^{2,3}$ \\ 'Department of Physical Education, College of Physical Education, Keimyung University, Daegu; ${ }^{2}$ KMF Co., Ltd., Daegu, ${ }^{3}$ Department of Food Science and Technology, \\ College of Natural Science, Keimyung University, Daegu, Korea
}

Background: It is important for older adults to prevent sarcopenia, but there are not currently effective methods to maintain or improve skeletal muscle function or immune function in this population. The goal of this study was to observe the effects of a 12-week resistance exercise program with fermented soybean supplementation on glucose tolerance and myokine expression in middle-aged obese rats in regard to body composition and immune senescence.

Methods: Subjects were randomly assigned to four groups: control (CON), soybean (SO), resistance exercise (RE), and soybean plus resistance exercise (SR). Resistance exercise involved ladder climbing, and soybean supplementation comprised $24 \%$ of total calories from soybean protein. Obesity was induced with 6 weeks of a high-fat diet, and then the group-specific procedures were conducted for the following 12 weeks.

Results: There were no significant differences between the groups in regard to daily dietary intake and body weight. The SO group had significantly higher intraperitoneal fat levels compared to the CON group $(P<0.05)$, and a significantly lower lean body mass $(P<0.05)$. The glycemic response of the SO group and SR group showed significantly higher improvements compared to the CON group and RE group $(P<0.05)$. The expression of myokines related to immune senescence was significantly lower in the SO group $(P<0.05)$.

Conclusion: Fermented soy beans helped improve glucose tolerance when it was applied with and without resistance exercise, with a significant reduction in the expression of inflammatory factors related to immune senescence in skeletal muscle.

Key words: Resistance training, Soybeans, Obesity, Senescence, Myokine

Received March 2, 2018

Reviewed May 16, 2018

Accepted July 18, 2018

${ }^{*}$ Corresponding author

Kijin Kim

https://orcid.org/0000-0002-3724-7758

Department of Physical Education, College of Physical Education, Keimyung University, 1095 Dalgubeol-daero, Dalseo-gu, Daegu 42601, Korea

Tel: +82-53-580-5256

Fax: +82-53-580-5314

E-mail: kjk744@kmu.ac.kr

\section{INTRODUCTION}

Aging is associated with loss of muscle mass or sarcopenia. ${ }^{1} \mathrm{Al}-$ though muscle loss is partially due to physical inactivity, reductions in muscle mass and strength are also observed in highly trained athletes as aging progresses. ${ }^{2}$ Appropriately planned exercise training can prevent or reduce sarcopenia, but even with identical amounts of exercise, its effects are lessened in middle-aged people compared to younger people. ${ }^{3}$ Moreover, an increase in intraperitoneal fat is generally observed during senescence, which causes overactivation of mammalian target of rapamycin, regulating cell growth, differentiation, and protein synthesis, and attenuating muscle growth. ${ }^{4}$ The combination of obesity and sarcopenia due to senescence can significantly increase one's mortality rate.

Copyright (c) 2018 Korean Society for the Study of Obesity

@) This is an Open Access article distributed under the terms of the Creative Commons Attribution Non-Commercial License (http://creativecommons.org/licenses/by-nc/4.0/) which permits unrestricted non-commercial use, distribution, and reproduction in any medium, provided the original work is properly cited. 
Immune senescence, centering on the reduction of acquired immune function, is not only related to a high mortality rate from all causes, including infectious diseases ${ }^{5}$, but is also sarcopenic obesity and characteristic body compositional changes. ${ }^{2}$ Increased amounts of inflammatory cytokines are observed in middle-aged people, and are highly related to reduced muscle mass and strength. ${ }^{6}$ Proinflammatory cytokines have been reported to increase the proteolysis of skeletal muscle in both in vitro and rodent models. ${ }^{6}$

Recently, the anti-inflammatory effects of exercise training have been described, along with reductions in intraperitoneal fat and toll-like receptor (TLR) expression on monocytes and macrophages. Moreover, many studies have examined the increased production and secretion of anti-inflammatory cytokines in contracted muscles. ${ }^{7}$ Myokines produced in contracting muscles, such as myostatin, leukemia inhibitory factor interleukin (IL) 6 family cytokine, IL-4, IL-6, IL-7, and IL-15, can regulate muscle hypertrophy and myodifferentiation. ${ }^{8}$ Brain-derived neurotrophic factor and IL-6 promote fat oxidation through 5 AMP-activated protein kinase activation ${ }^{9}$, and IL-8 aids in blood vessel differentiation after exercise. ${ }^{10}$ Moreover, IL-4 and IL-10, which have mainly anti-inflammatory effects, can increase myogenesis. ${ }^{11}$ The production and secretion of myokines provide a mechanism by which exercise maintains and improves health, and exercise-induced muscle hypertrophy or reduced myoatrophy in middle-age can be viewed as the result of various myokine interactions within skeletal muscles.

Resistance exercise has been widely suggested as a means to prevent sarcopenic obesity, and increase muscle mass, strength, and function. ${ }^{12,13}$ However, in reality, there are limits to how much exercise one can do to maintain skeletal muscle function in a state of sarcopenic obesity due to senescence. Nutritional interventions, such as specialized dietary regimes, may provide synergistic effects with exercise. Increased protein intake along with resistance exercise can promote positive changes in body composition by increasing muscle protein synthesis and lean body mass. ${ }^{14}$ However, the supplemental protein used in previous studies have generally been animal proteins, which are less effective in older adults who have decreased digestive and absorptive capabilities. In addition, the expense of these protein sources may prohibit increased consumption.

Soybean is a widely used ingredient in various Korean fermented products, such as cheonggukjang (fermented soybean paste), doenjang (fermented soybean paste), and gochujang (red pepper paste). Fermented soybean pastes are produced by fermenting the beans with Bacillus subtilis for a relatively short period of time, during which various enzymes, microorganisms, and bioactive compounds not found in unfermented soybeans are produced..$^{15}$ Cheonggukjang, which is the most representative form of fermented soybeans, may have antiobesogenic, antidiabetic, and anti-inflammatory effects that could positively influence chronic diseases in the body. According to clinical studies, consuming cheonggukjang significantly reduces intraperitoneal fat and apolipoprotein $\mathrm{B} /$ A1 levels. ${ }^{16}$ Also, diet-induced obese C57BL/6J mice showed improved body weight, epididymal fat accumulation, total serum cholesterol, and low-density lipoprotein cholesterol after 9 weeks of cheonggukjang consumption. ${ }^{17}$ By analyzing the anti-inflammatory activities of fermented soybeans in a type 1 hypersensitivity and arachidonic acid-induced ear edema model, fermented soybean treatment reduced cutaneous anaphylaxis. Furthermore, ethanol extracts of fermented soybeans were found to significantly increase cell viability by inhibiting apoptosis. ${ }^{18}$

Based on the previous studies, we hypothesized that the antiobesogenic effects of fermented soybeans and muscle hypertrophy effects of resistance exercise would effectively increase the immune functions of those with sarcopenic obesity due to senescence. Therefore, this study examined the effects of 12 weeks of resistance exercise and fermented soybean consumption on obese middleaged Wistar rats, with the aim of generating preclinic evidence that can be used to increase the quality of life of older adults suffering from sarcopenic obesity and low immune function.

\section{METHODS}

\section{Subjects}

First, 50-week-old male Wistar rats $(\mathrm{n}=40)$ were purchased. After spending 1 week adjusting to their new environment, obesity was induced by feeding them a high-fat diet ( $45 \%$ calories from fat) for 8 weeks. Then, rats were randomly assigned to four groups: control (CON), soybean (SO), resistance exercise (RE), and soybean plus resistance exercise (SR). Two Wistar rats were housed per cage, and they were fed ad libitum throughout the 12-week 
treatment period. All rats were individually bred during the breeding period, and the day-night cycle was 12 hours. Temperature and relative humidity were maintained around $21^{\circ} \mathrm{C} \pm 1{ }^{\circ} \mathrm{C}$ and $60 \%$, respectively. The study design was approved by the Animal Experiment Ethics Committee of Daegu Technopark BioHealth Convergence Center (BHCC-IACUC-2016-02).

\section{Procedures}

\section{Dietary methods}

Throughout the experimental period, rats were fed as described in Jung and Kim. ${ }^{19}$ The dietary regime consisted of a high-fat diet (35\% carbohydrates, 45\% fat, 24\% protein, \#D12451; Research Diets Inc., New Brunswick, NJ, USA). During the 12-week experimental period, rats consumed diets that differed solely in the type of protein. The $\mathrm{CON}$ and RE groups received standard casein protein, while the SO and SR groups received fermented soybean protein instead, which was consumed as pellets. Fermented soybeans were made from domestically produced soybeans with help from the Department of Food Science and Technology, Keimyung University. A B. subtilis strain isolated from doenjang was used for fermentation after precultivation at $37^{\circ} \mathrm{C}$ for 24 hours, by inoculation at $1 \%$ in lysogeny broth (10\% Bacto-tryptone, $5 \%$ yeast extract, 10\% sodium chloride; Duchefa Biochemie, Haarlem, the Netherlands), and culturing for 9 hours. Cultured B. subtilis DJI was centrifuged at $4^{\circ} \mathrm{C}$ and $9,950 \times g$ for 15 minutes and rinsed twice with sterilized deionized water. The soybeans were soaked in three times their volume of water for 20 hours after being selected and rinsed, steamed in high pressure sterilizers for 50 minutes, and cooled to $40^{\circ} \mathrm{C}$. Initial batches were inoculated with the obtained $1 \%$ enrichment culture $(\mathrm{v} / \mathrm{w})$ and fermented at $37^{\circ} \mathrm{C}$ for 11 hours. Selected soybeans $(1 \mathrm{~kg})$ were soaked at $20^{\circ} \mathrm{C}$ for 20 hours, steamed at $121^{\circ} \mathrm{C}$ for 40 minutes, and the produced pressure-steamed fermented beans were freeze-dried, pulverized into powder, vacuumpacked, and stored at $-80^{\circ} \mathrm{C}$ until use.

\section{Resistance exercise protocol}

The resistance exercise protocol comprised ladder climbing, and was developed by partially modifying the methods reported by Jung and Kim. ${ }^{19}$ For the 1st week, the rats practiced climbing on a $1.5 \mathrm{~m}$ ladder at a $80^{\circ}$ incline without weights on their tails to habit- uate them to the exercise. Subsequently, weights of $25 \%-45 \%$ of their body weight were attached to the rats' tails for ladder-climbing training, and the weights and the number of repetitions was incrementally increased. From the second week until the completion of the exercise program, set 1 was conducted with weights of $65 \%$ of body weight; sets 2 and 3 with weights of $75 \%$ of body weight; sets 4 and 5 with weights of $85 \%-90 \%$ of body weight; and sets $6-8$ with weights of $100 \%-110 \%$ of body weight. Each set involved eight repetitions and was separated from the next by 2 minutes of rest. The exercise was conducted three times per week.

\section{Oral glucose tolerance test}

After 10 weeks of treatment, the rats rested for 48 hours to eliminate the effects of the last bout of exercise and were fasted. Blood was collected at rest from the tails and $1 \mathrm{~g} / \mathrm{kg}$ of $50 \%$ glucose was orally administered. Then, $400 \mu \mathrm{L}$ of blood was collected after 15 , 30,60 , and 120 minutes. The collected blood was treated with an anticoagulant (50 $\mu \mathrm{L}$ heparin), and plasma was extracted through centrifugation $(1,500 \times g, 15$ minutes $)$ and stored at $-80^{\circ} \mathrm{C}$ until further analysis. Blood glucose was measured through enzymatic analysis using an automated blood glucose monitor (YSI 2300; YSI Life Sciences, Springfield, NJ, USA). The rats were administered a $2.5 \mathrm{~mL}$ subcutaneous injection of $0.9 \%$ saline solution to replenish their lost plasma after blood collection. The next exercise treatment was conducted after a 3-day recovery period.

\section{Tissue extraction and blood sampling}

After 12 weeks of treatment, the animals were allowed to rest for 48 hours to avoid the "last-bout" effect. Subsequently, they were fasted for 12 hours and anesthetized with ketamine $(70 \mathrm{mg} / \mathrm{kg}$ body weight; Virbac, Seoul, Korea) and xylazine $(10 \mathrm{~mL} / \mathrm{kg}$; Bayer, Seoul, Korea). First, the abdominal cavity was opened to collect blood from the abdominal artery. Plantaris muscles and intraperitoneal fat (epididymal, mesenteric, and retroperitoneal fat pads) were subsequently extracted and weighed, flash frozen, and stored at $-80^{\circ} \mathrm{C}$ until analysis.

\section{Measured variables and analytical methods Measuring body mass and dietary intake}

During the 12-week research period, body mass and dietary in- 
take were measured up to the first decimal place every other day at 9 AM using a scale (PJ6; Mettler, Giessen, Germany).

\section{Body composition}

The rats were anesthetized through intraperitoneal administration of $70 \mathrm{mg} / \mathrm{kg}$ ketamine and $10 \mathrm{mg} / \mathrm{kg}$ of xylazine (both from JW Pharmaceutical Corp., Seoul, Korea), and their intraperitoneal lean body mass and fat mass were measured using dual energy $\mathrm{X}$ ray absorptiometry (DSC-3000; Aloka, Tokyo, Japan) and measuring the weight of extracted organs.

\section{Western blotting}

The extracted plantaris muscle was homogenized in an ice-cold buffer containing $250 \mathrm{mM}$ sucrose, $10 \mathrm{mM}$ HEPES/ $1 \mathrm{mM}$ ethylenediaminetetraacetic acid (EDTA; pH 7.4), 1 mM Pefabloc (Roche, Basel, Switzerland), $1 \mathrm{mM}$ EDTA, $1 \mathrm{mM} \mathrm{NaF,} 1 \mathrm{~g} / \mathrm{mL}$ aprotinin, $1 \mathrm{~g} / \mathrm{mL}$ leupeptin, $1 \mathrm{~g} / \mathrm{mL}$ pepstatin, $0.1 \mathrm{mM} \mathrm{bpV(phen),} \mathrm{and}$ $2 \mathrm{mg} / \mathrm{mL}$ glycerophosphate. The homogenized sample was freezethawed three times and centrifuged at $700 \times g$ for 10 minutes. The supernatant was collected and proteins were quantified with a Lowry et al..s ${ }^{20}$ assay. Samples were mixed with Laemmli buffer, loaded onto an sodium dodecyl sulfate-polyacrylamide gel, and electrophoresed. Proteins were then transferred onto a nitrocellulose membrane, which was subsequently blocked for 60 minutes with 5\% nonfat dry milk in tris-buffered saline+0.1\% Tween 10 (TBST; pH 7.5). After being washed with TBST, the membrane was incubated overnight with a primary antibody against one of the following proteins: TLR4, myeloid differentiation primary response 88 (MyoD88), IL-6, IL-15 (Santa Cruz Biotechnology, Santa Cruz, CA, USA). The membrane was then washed with TBST and exposed to secondary antibodies for 60 minutes. ECL (Genekhan Scientific, St. Louis, MO, USA) was used for visualization, and SigmaGel (Jandel Scientific Corp., Erkrath, Germany) was used to control for relative band intensity.

\section{Statistical analysis}

The results of each measured variable represent the mean and standard error, and statistical analysis was conducted using the SPSS version 10.0 (SPSS Inc., Chicago, IL, USA). One-way analysis of variance (ANOVA) was conducted to verify the differences between the groups after 12 weeks of treatment, and two-way ANOVA was conducted to verify these differences at different times. Post-hoc verification was conducted using Tukey test, and statistical significance was set at $P<0.05$.

\section{RESULTS}

\section{Dietary intake and body mass}

After 12 weeks of treatment, the daily dietary intakes of obese middle-aged Wistar rats did not significantly differ between groups although the weights did gradually increase over time (Fig. 1A). Body weight was higher in the CON group compared to the other groups, but there was no significant difference (Fig. 1B).

\section{Body composition}

Intraperitoneal lean body mass was significantly lower in the $\mathrm{SO}$ group compared to the CON group $(P<0.05)$, while the intraperitoneal fat mass was significantly higher than in the other three groups $(P<0.05)$. However, there were no significant differences in intraperitoneal fat mass of the RE and SR groups compared to
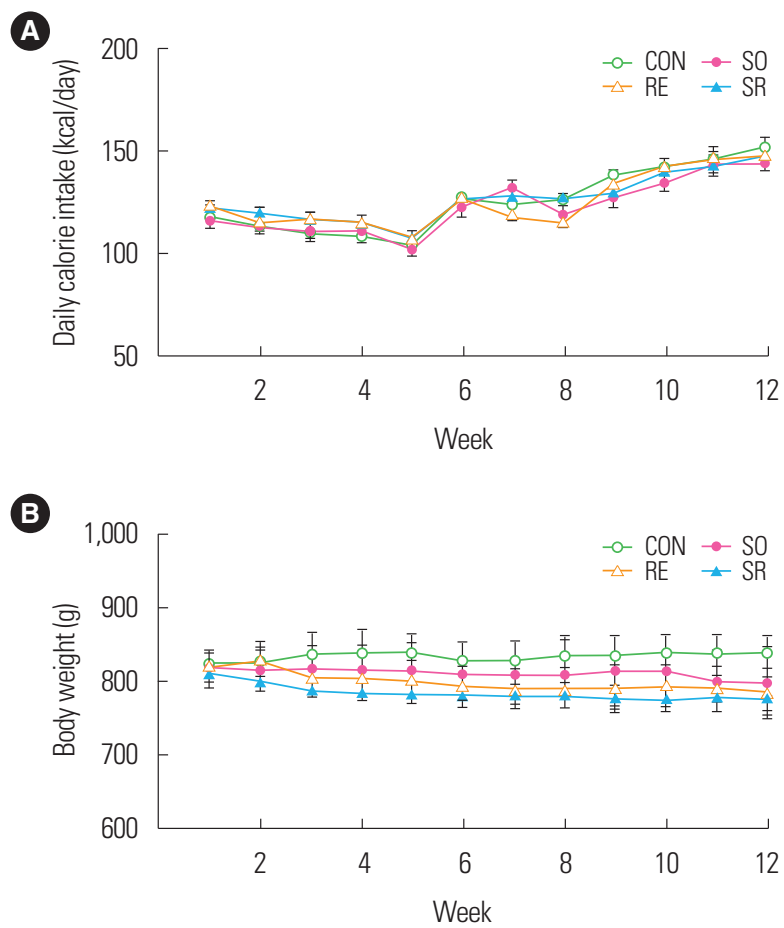

Figure 1. Changes in daily calorie intake (A) and body weight (B) of the groups. CON, control; SO, soybean; RE, resistance exercise; SR, soybean plus resistance exercise. 
A

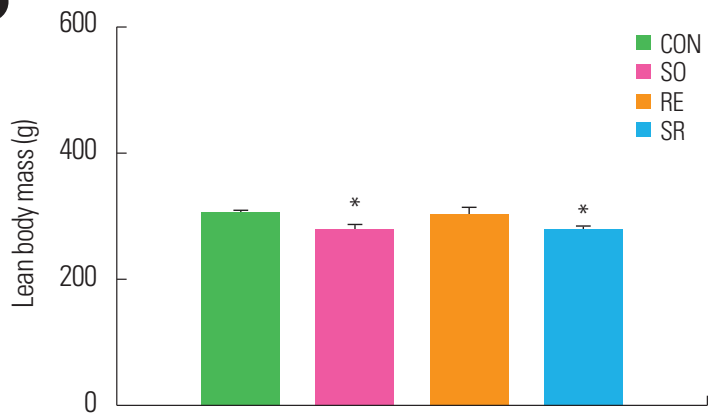

B

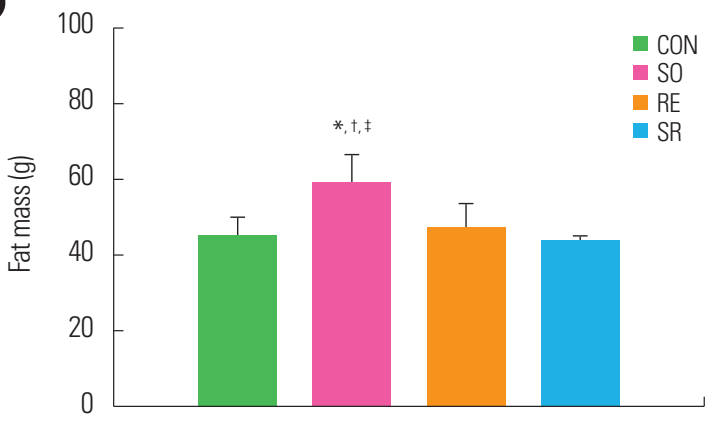

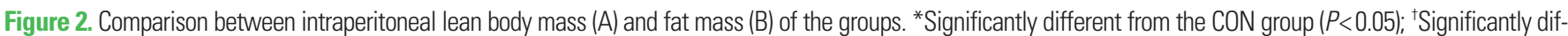
ferent from the RE group $(P<0.05)$; ' Significantly different from the SR group $(P<0.05)$. CON, control; SO, soybean; RE, resistance exercise; SR, soybean plus resistance exercise.

the CON group (Fig. 2).

\section{Muscle mass}

After 12 weeks of treatment, there were no significant differences in the weight of the plantaris muscle, a lower extremity fast-twitch muscle, between the SO, RE, and SR groups compared to the CON group (Fig. 3).

\section{Oral glucose tolerance test}

An oral glucose tolerance test was conducted to observe the effects of 12 weeks of fermented soybean consumption and resistance exercise on the glucose tolerance of obese middle-aged Wistar rats. The blood glucose responses of the SO and SR groups were found to be significantly improved compared to those of the CON and RE groups $(P<0.05)$ (Fig. 4).

\section{Myokine expression}

To investigate the effects of 12 weeks of fermented soybean consumption and resistance exercise on the expression of myokines related to the immune senescence of skeletal muscles, the plantaris muscle was excised and its myokines were quantitatively analyzed. TLR4 protein expression significantly decreased in the $\mathrm{SO}$ and SR groups compared to the CON group $(P<0.05)$ (Fig. 5A). The expressions of MyoD88 and IL-6 were significantly lower in the SO group than in the other three groups $(P<0.05)$ (Fig. 5B, C). The expression of IL-15 did not significantly differ between the groups (Fig. 5D).

\section{DISCUSSION}

The aim of this study was to investigate the effects of long-term resistance exercise and consumption of fermented soybeans on body composition, glucose tolerance, and expression of immune senescence-related myokines. Although resistance exercise training did not have a significant influence on body composition, consumption of fermented soybeans reduced the lean body mass and significantly increased the intraperitoneal fat mass of obese middleaged Wistar rats. The nonsignificant effect of resistance exercise training suggested the need of additional consideration on the focus of exercise duration and details of the ladder-climbing exercise. The SO and SR groups, which consumed fermented soybeans, showed significantly reduced blood glucose responses during oral glucose tolerance tests compared to the RE group. Kim et al. ${ }^{21}$ conducted a 4-week-long dietary study on 5-week-old male Wistar rats, examining the effects of soybean, cheonggukjang, and doenjang powder consumption on changes in blood glucose and serum lipid levels in rats with streptozotocin-induced diabetes. Rats that consumed cheonggukjang had reduced body mass and blood glucose concentrations compared to control rats. Furthermore, various studies have reported that the consumption of fermented soybeans has antidiabetic effects, improving blood glucose concentrations in diabetic and obese Wistar rats. ${ }^{22,23}$ In the majority of these studies, both body fat reduction and blood glucose improvement were observed after consuming fermented soybeans. However, a reduction in body mass was not observed in this study. Rather, intraperitoneal fat increased and lean body mass decreased. Li et al. ${ }^{24}$ fed $5 \%$ fermented soybean (cheonggukjang) powder to $\mathrm{db} / \mathrm{db}$ mice for 6 


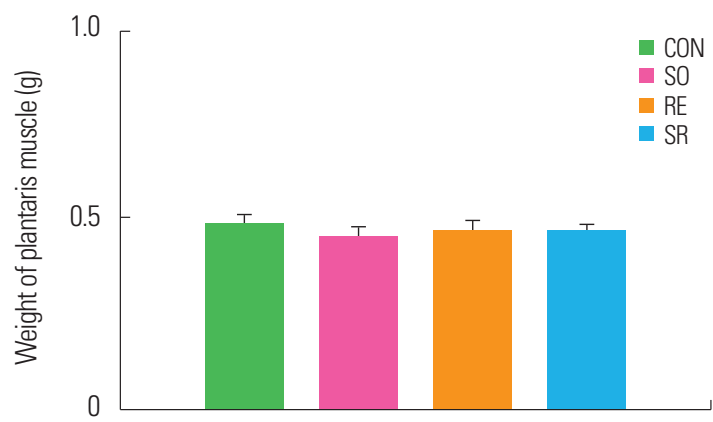

Figure 3. Differences in plantaris muscle mass between groups. CON, control; SO, soybean; RE, resistance exercise; SR, soybean plus resistance exercise.

weeks, and reported that the blood glucose and hemoglobin contents were significantly reduced, although there was no influence on the blood insulin concentration. These results were thought to be due to improved high glucose and insulin tolerance by increasing the functions of pancreatic $\beta$-cells of $\mathrm{db} / \mathrm{db}$ mice. Moreover, Liu et al. ${ }^{25}$ reported that genistein, which comprises approximately $70 \%$ of fermented soybeans, increases insulin production in the mouse pancreas after glucose stimulation. The results of this study suggest that blood glucose improvements after consuming fermented soybeans are not due to changes in body composition, but to improvements in blood glucose regulation. The results of this study (i.e., no significant difference in fat mass or body weight in the SO group) might be different from those of previous studies because of the similar increase in the daily dietary intakes with CON group and the complex factor of age; however, additional studies will be required to verify this and elucidate the related mechanisms.

Long-term consumption of fermented beans significantly reduced the expression of myokines related to immune senescence. Senescence and obesity increase the expression of TLRs, increasing their downstream regulators, MyoD88 and IL-6, and decreasing immune function by causing inflammation. These phenomena have been reported to increase proteolysis in skeletal muscle both in vitro and in rodents. ${ }^{26,27}$ In this study, TLR4 expression decreased significantly with soybean consumption, and MyoD88 and IL-6 had significantly lower protein expression in the SO group. TLR4 decreased in both SO and SR groups, whereas myoD88 only differed in the SO group. This study could not explain why the decrease in myoD88 in the SR group is weakened compared to the

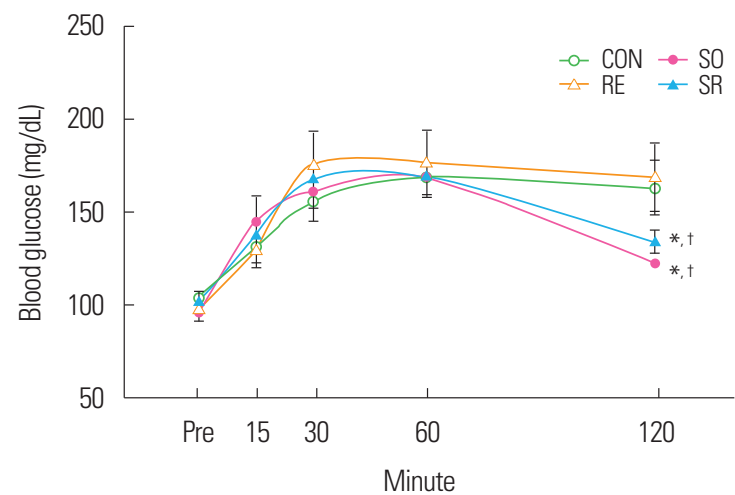

Figure 4. Blood glucose responses during the oral glucose tolerance test. *Significantly different from the CON group $(P<0.05)$; 'Significantly different from the RE group $(P<0.05)$. CON, control; SO, soybean; RE, resistance exercise; SR, soybean plus resistance exercise.

SO group. Further study will be required to verify the myoD88-related mechanisms.

Solerte et al. ${ }^{28}$ analyzed insulin-like growth factor 1 and tumor necrosis factor- $\alpha$ (TNF- $\alpha)$ concentrations based on protein intake, and reported an anabolic effect of reduced TNF- $\alpha$ expression. Moreover, Nam et al. ${ }^{29}$ argued that TNF- $\alpha$ and C-C motif chemokine ligand 2 (CCL2) are activated in the adipose tissues of highfat diet-fed mice, along with oxidative stress markers, and these proinflammatory cytokines are inhibited after consuming doenjang. Consuming soybeans activates anti-inflammatory markers, including adiponectin, making it very effective in relieving obesity-induced inflammation and oxidative stress. Moreover, Oh et al..$^{30}$ reported inhibitory effects on the inflammatory markers IL- $1 \beta$ and IL-6 after feeding seaweed that contained sufficient amounts of protein to high-fat diet-induced obese mice. Han et al. ${ }^{31}$ reported that soy leaf extracts are effective at reducing proinflammatory cytokines levels in blood vessels, including TNF- $\alpha$, IL-6, IL-1 $\beta$, CCL2, vascular cell adhesion molecule 1 , and intercellular adhesion molecule 1. Moreover, Mercer et al. ${ }^{32}$ reported that soy protein isolates have inhibitory effects on high-fat diet-induced hepatoma proliferation in mice, by reducing IL- 6 and TNF- $\alpha$ expression. Consistent with these previous studies, our results indicate that consumption of fermented soybeans is effective at preventing inflammation and increasing immune system function during senescence and obesity.

IL-15 acts as an anabolic factor for skeletal muscle growth. ${ }^{33,34}$ It positively affects immune function by functioning as a speed regu- 
A

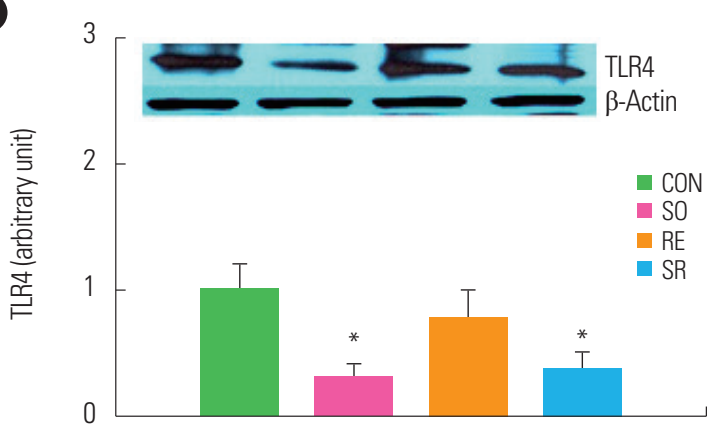

C

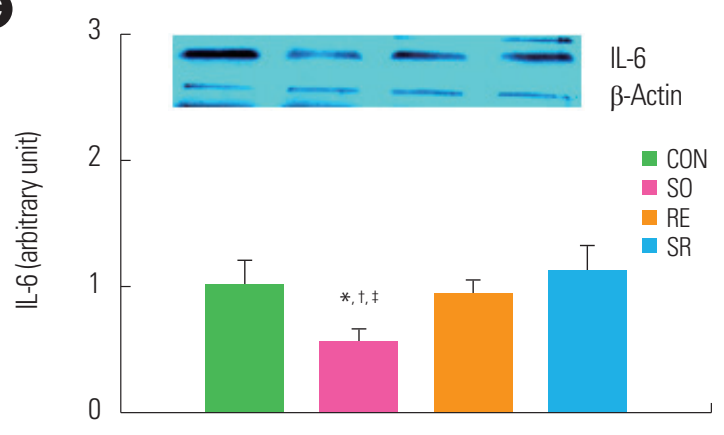

B

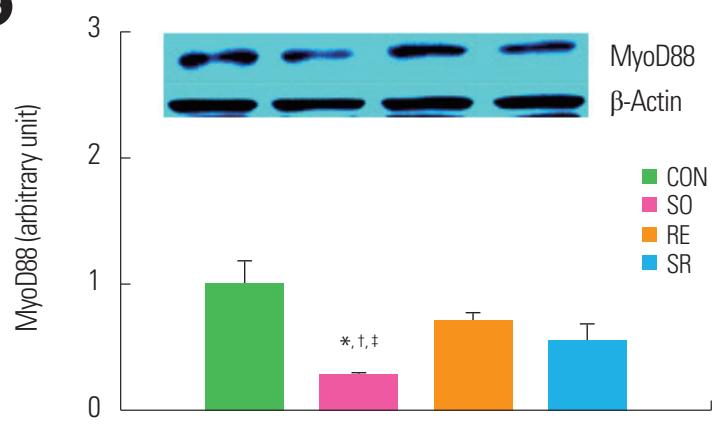

(D)

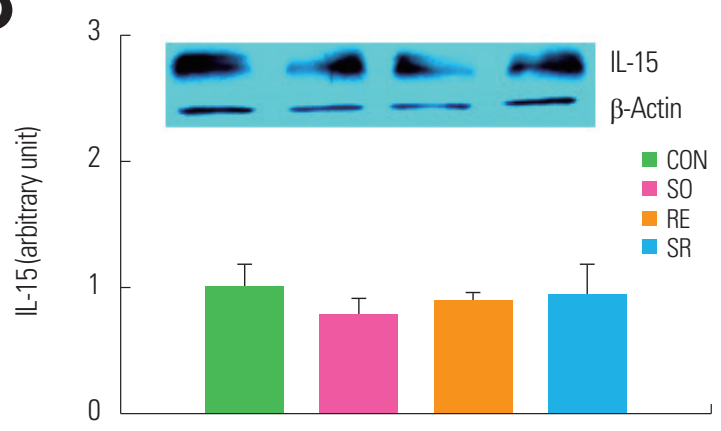

Figure 5. Comparison of toll-like receptor 4 (TLR4, A), myeloid differentiation primary response 88 (MyoD88, B), interleukin (IL)-6 (C), and IL-15 (D) protein expression. *Significantly different from the CON group $(P<0.05)$; 'Significantly different from the RE group $(P<0.05)$; 'Significantly different from the SR group $(P<0.05)$. CON, control; SO, soybean; RE, resistance exercise; SR, soybean plus resistance exercise.

latory factor for natural killer cells, increasing their number and function. ${ }^{35,36}$ It has been predicted that IL-15 protein levels in muscle and blood plasma significantly decrease during senescence, while dietary treatments such as limited calorie intake increase IL15 expression, and resistance exercise $\mathrm{e}^{37}$ and aerobic exercise $\mathrm{e}^{38}$ have positive effects on IL-15 expression. This suggests that consumption of fermented soybeans and resistance exercise should significantly increase IL-15 expression. However, there were no significant differences in IL-15 levels between the different groups. It should be noted that most previous studies analyzing the effects of exercise on IL-15 expression were conducted in healthy adults ${ }^{39,40}$, and it is unclear whether the expression level was continuous. ${ }^{38}$ Therefore, it is suspected that differences in research subjects and analysis times may have caused this difference. Further study will be required to suggest a definite conclusion related to IL-15 expression. In summary, although our results do not have detailed data on muscle function, a complete array of myokines, and the typical phenotype of the RE group, we found that 12 weeks of resistance exercise and fermented bean consumption for obese middle-aged Wistar rats did not significantly improve body composition, but improved glucose tolerance and significantly reduced the levels of inflammatory factors related to immune senescence in skeletal muscle. This suggests that the consumption of fermented soybeans in the states of obesity and senescence can help prevent inflammation and increase immune function.

\section{CONFLICTS OF INTEREST}

The authors declare no conflict of interest.

\section{ACKNOWLEDGMENTS}

This study was supported by 2016 Moonsuk Research Grant from Korean Society for the Study of Obesity (KSSO).

\section{REFERENCES}

1. Roubenoff R. Sarcopenic obesity: does muscle loss cause fat gain? Lessons from rheumatoid arthritis and osteoarthritis. Ann N Y Acad Sci 2000;904:553-7. 
2. Pedersen BK. Anti-inflammation: just another word for antiageing? J Physiol 2009;587(Pt 23):5515.

3. Burks TN, Cohn RD. One size may not fit all: anti-aging therapies and sarcopenia. Aging (Albany NY) 2011;3:1142-53.

4. Williamson DL. Normalizing a hyperactive mTOR initiates muscle growth during obesity. Aging (Albany NY) 2011;3:83-4.

5. Wikby A, Ferguson F, Forsey R, Thompson J, Strindhall J, Löfgren S, et al. An immune risk phenotype, cognitive impairment, and survival in very late life: impact of allostatic load in Swedish octogenarian and nonagenarian humans. J Gerontol A Biol Sci Med Sci 2005;60:556-65.

6. Tilg H, Moschen AR. Inflammatory mechanisms in the regulation of insulin resistance. Mol Med 2008;14:222-31.

7. Carpenter KC, Strohacker K, Breslin WL, Lowder TW, Agha $\mathrm{NH}, \mathrm{McFarlin} \mathrm{BK}$. Effects of exercise on weight loss and monocytes in obese mice. Comp Med 2012;62:21-6.

8. Stránská Z, Svačina Š. Myokines: muscle tissue hormones. Vnitr Lek 2015;61:365-8.

9. Pedersen BK, Febbraio MA. Muscles, exercise and obesity: skeletal muscle as a secretory organ. Nat Rev Endocrinol 2012; $8: 457-65$.

10. Yeo NH, Woo J, Shin KO, Park JY, Kang S. The effects of different exercise intensity on myokine and angiogenesis factors. J Sports Med Phys Fitness 2012;52:448-54.

11. Tidball JG, Villalta SA. Regulatory interactions between muscle and the immune system during muscle regeneration. Am J Physiol Regul Integr Comp Physiol 2010;298:R1173-87.

12. Molanouri Shamsi M, Hassan ZH, Gharakhanlou R, Quinn LS, Azadmanesh K, Baghersad L, et al. Expression of interleukin-15 and inflammatory cytokines in skeletal muscles of STZ-induced diabetic rats: effect of resistance exercise training. Endocrine 2014;46:60-9.

13. Talebi-Garakani E, Safarzade A. Resistance training decreases serum inflammatory markers in diabetic rats. Endocrine 2013; 43:564-70.

14. Frestedt JL, Zenk JL, Kuskowski MA, Ward LS, Bastian ED. A whey-protein supplement increases fat loss and spares lean muscle in obese subjects: a randomized human clinical study. Nutr Metab (Lond) 2008;5:8.

15. Lee YB, Lee HJ, Won MH, Hwang IK, Kang TC, Lee JY, et al.
Soy isoflavones improve spatial delayed matching-to-place performance and reduce cholinergic neuron loss in elderly male rats. J Nutr 2004;134:1827-31.

16. Back HI, Kim SR, Yang JA, Kim MG, Chae SW, Cha YS. Effects of chungkookjang supplementation on obesity and atherosclerotic indices in overweight/obese subjects: a 12-week, randomized, double-blind, placebo-controlled clinical trial. J Med Food 2011;14:532-7.

17. Soh J, Kwon DY, Cha YS. Hepatic gene expression profiles are altered by dietary unsalted Korean fermented soybean (chongkukjang) consumption in mice with diet-induced obesity. J Nutr Metab 2011;2011:260214.

18. Kim HB, Lee HS, Kim SJ, Yoo HJ, Hwang JS, Chen G, et al. Ethanol extract of fermented soybean, chungkookjang, inhibits the apoptosis of mouse spleen, and thymus cells. J Microbiol 2007;45:256-61.

19. Jung SR, Kim KJ. Effects of resistance exercise training and fermented soybean treatment on activity of mTOR and FoxO in skeletal muscle of middle aged obese rats. Korean J Physic Educ 2017;56:509-18.

20.Lowry OH, Rosebrough NJ, Farr AL, Randall RJ. Protein measurement with the Folin phenol reagent. J Biol Chem 1951; 193:265-75.

21. Kim AR, Lee JJ, Cha SS, Chang HC, Lee MY. Effect of soybeans, chungkukjang, and doenjang on blood glucose and serum lipid profile in streptozotocin-induced diabetic rats. J Kor Soc Food Sci Nutr 2012;41:621-9.

22. Lim KH, Han JH, Lee JY, Park YS, Cho YS, Kang KD, et al. Assessment of antidiabetogenic potential of fermented soybean extracts in streptozotocin-induced diabetic rat. Food Chem Toxicol 2012;50:3941-8.

23. Park S, Kim DS, Kim JH, Kim JS, Kim HJ. Glyceollin-containing fermented soybeans improve glucose homeostasis in diabetic mice. Nutrition 2012;28:204-11.

24. Li H, Ji HS, Kang JH, Shin DH, Park HY, Choi MS, et al. Soy leaf extract containing kaempferol glycosides and pheophorbides improves glucose homeostasis by enhancing pancreatic $\beta$-cell function and suppressing hepatic lipid accumulation in db/db mice. J Agric Food Chem 2015;63:7198-210.

25. Liu D, Zhen W, Yang Z, Carter JD, Si H, Reynolds KA. Genis- 
tein acutely stimulates insulin secretion in pancreatic beta-cells through a cAMP-dependent protein kinase pathway. Diabetes 2006;55:1043-50.

26. Larbi A, Franceschi C, Mazzatti D, Solana R, Wikby A, Pawelec G. Aging of the immune system as a prognostic factor for human longevity. Physiology (Bethesda) 2008;23:64-74.

27. Wikby A, Johansson B, Olsson J, Löfgren S, Nilsson BO, Ferguson F. Expansions of peripheral blood CD8 T-lymphocyte subpopulations and an association with cytomegalovirus seropositivity in the elderly: the Swedish NONA immune study. Exp Gerontol 2002;37:445-53.

28. Solerte SB, Gazzaruso C, Bonacasa R, Rondanelli M, Zamboni M, Basso C, et al. Nutritional supplements with oral amino acid mixtures increases whole-body lean mass and insulin sensitivity in elderly subjects with sarcopenia. Am J Cardiol 2008; 101(11A):69E-77E.

29. Nam YR, Won SB, Chung YS, Kwak CS, Kwon YH. Inhibitory effects of Doenjang, Korean traditional fermented soybean paste, on oxidative stress and inflammation in adipose tissue of mice fed a high-fat diet. Nutr Res Pract 2015;9:235-41.

30. Oh JH, Kim J, Lee Y. Anti-inflammatory and anti-diabetic effects of brown seaweeds in high-fat diet-induced obese mice. Nutr Res Pract 2016;10:42-8.

31. Han JM, Li H, Cho MH, Baek SH, Lee CH, Park HY, et al. Soy-leaf extract exerts atheroprotective effects via modulation of Krüppel-like factor 2 and adhesion molecules. Int J Mol Sci 2017;18:E373.

32. Mercer KE, Pulliam CF, Pedersen KB, Hennings L, Ronis MJ. Soy protein isolate inhibits hepatic tumor promotion in mice fed a high-fat liquid diet. Exp Biol Med (Maywood) 2017;242: $635-44$.
33. Argilés JM, López-Soriano J, Almendro V, Busquets S, LópezSoriano FJ. Cross-talk between skeletal muscle and adipose tissue: a link with obesity? Med Res Rev 2005;25:49-65.

34. Furmanczyk PS, Quinn LS. Interleukin-15 increases myosin accretion in human skeletal myogenic cultures. Cell Biol Int 2003;27:845-51.

35. Bouchaud G, Garrigue-Antar L, Solé V, Quéméner A, Boublik Y, Mortier E, et al. The exon-3-encoded domain of IL-15ralpha contributes to IL-15 high-affinity binding and is crucial for the IL-15 antagonistic effect of soluble IL-15Ralpha. J Mol Biol 2008;382:1-12.

36. Waldmann TA, Tagaya Y. The multifaceted regulation of interleukin-15 expression and the role of this cytokine in NK cell differentiation and host response to intracellular pathogens. Annu Rev Immunol 1999;17:19-49.

37. Riechman SE, Balasekaran G, Roth SM, Ferrell RE. Association of interleukin-15 protein and interleukin-15 receptor genetic variation with resistance exercise training responses. J Appl Physiol (1985) 2004;97:2214-9.

38. Tamura Y, Watanabe K, Kantani T, Hayashi J, Ishida N, Kaneki M. Upregulation of circulating IL-15 by treadmill running in healthy individuals: is IL-15 an endocrine mediator of the beneficial effects of endurance exercise? Endocr J 2011;58: 211-5.

39. Barra NG, Reid S, MacKenzie R, Werstuck G, Trigatti BL, Richards C, et al. Interleukin-15 contributes to the regulation of murine adipose tissue and human adipocytes. Obesity (Silver Spring) 2010;18:1601-7.

40. Carbó N, López-Soriano J, Costelli P, Busquets S, Alvarez B, Baccino FM, et al. Interleukin-15 antagonizes muscle protein waste in tumour-bearing rats. Br J Cancer 2000;83:526-31. 